\title{
Évolution de la production laitière des vaches à la rentrée à l'étable : influence de la nature de la ration de base et de la méthode de complémentation en aliment concentré
}

\author{
J.B. Coulon 1 , P. D'Hour ${ }^{2}$ et J. Rouel 3 \\ avec la collaboration technique de E. Albaret ${ }^{2}$ et R. Lefaivre ${ }^{1}$
}

1 INRA, laboratoire de la lactation, 63122 Ceyrat;
2 INRA, domaine expérimental, 15190 Marcenat;
3 INRA, domaine expérimental, 63210 Orcival, France

(reçu le17 mars 1989, accepté le 24 juin 1989)

\begin{abstract}
Résumé - A la rentrée à l'étable en stabulation entravée, 2 essais ont été réalisés, avec des vaches multipares en fin de lactation (huitième mois en moyenne). Dans l'essai 1,16 vaches ont reçu à volonté, dès leur rentrée à l'étable, un régime composé soit d'ensilage de maïs (lot EM), soit d'ensilage d'herbe (lot EH). Ces 2 régimes ont été complémentés normalement en aliment concentré (au-dessus d'une production de $13 \mathrm{~kg} / \mathrm{j}$ de lait). Dans l'essai 2,24 vaches ont reçu, dès leur rentrée à l'étable, un régime à base de foin complémenté en concentré soit normalement (au-dessus d'une production de $8 \mathrm{~kg} / \mathrm{j}$ de lait), soit avec un apport supplémentaire de $28 \mathrm{~kg}$ de concentré réparti pendant les 2 premières semaines de stabulation (lot F22) ou uniquement pendant la première semaine (lot F40). Dans les 2 essais, les quantités de fourrages ingérées ont augmenté fortement et rapidement à la rentrée à l'étable. Dans l'essai 1 , elles ont été en moyenne supérieures de $2 \mathrm{~kg} \mathrm{MS} / \mathrm{j}$ dans le lot EM par rapport au lot EH. La production laitière a diminué plus fortement dans le lot EH $(-3,8$ $\mathrm{kg} / \mathrm{j}$ entre la dernière semaine de pâturage et la première semaine de stabulation) que dans le lot EM $(-2,9 \mathrm{~kg} / \mathrm{j}, P<0,01)$. Dans l'essai 2, elle a diminué d'autant moins fortement que les apports de concentré en première semaine de stabulation étaient plus importants (respectivement $-1,9,-1,2$ et $-0,6 \mathrm{~kg} / \mathrm{j}$ dans les lots F00, F22 et F40). Au cours des essais, les animaux ont gagné entre 0,9 et 1,1 $\mathrm{kg}$ de masse corporelle par jour suivant les lots. Les différentes origines possibles de ces évolutions sont discutées.
\end{abstract}

vache laitière - rentrée à l'étable — composition du lait — quantités ingérées

Summary - Dairy cow performance at stabling : influence of the type of roughage and method of concentrate supplementation. Two trials were made at stabling with multiparous dairy cows in late lactation (8th month on average). In trial 1, as soon as they were stabled, 16 cows were given a diet ad libitum composed either of maize silage (group EM) or grass silage (group EH). These 2 diets were normally supplemented with concentrate (above $13 \mathrm{~kg}$ milk/d). In trial 2, as soon as they were stabled, 24 cows were given a hay diet ad libitum supplemented with concentrate, either at a normal rate (above $8 \mathrm{~kg}$ milk/d) [group F00] or with an additional amount of $28 \mathrm{~kg}$ concentrate. The latter was offered during the first 2 weeks of stabling (2 kg/d, group F22) or only during the first week (4 kg/d, group F40). In trial 1 and 2, roughage dry matter intake increased largely and rap- 
idly at stabling. In trials 1, it was higher in group EM than in group $E H(+2 \mathrm{~kg} \mathrm{DM} / \mathrm{d}$, on an average). Milk production decrease was greater in group EH $(-3,8 \mathrm{~kg} / \mathrm{d}$ between the last week out-doors) than in group EM $(-2.9 \mathrm{~kg} / \mathrm{d}, \mathrm{P}<0.01)$. In trial 2, the higher the concentrate supply during the first week indoors, the lower the decrease in milk production $(-1.9,-1.2$ and $-0.6 \mathrm{~kg} / \mathrm{d}$ in group F00, F22 and F40, respectively). During the trials, cows gained between 0.9 and $1.1 \mathrm{~kg}$ body weight per day. The possible causes of these changes are discussed.

dairy cow - stabling - milk composition - intake

\section{INTRODUCTION}

La rentrée à l'étable à l'automne, faisant suite à une période de pâturage, est une période délicate pour les vaches laitières en production. Simultanément à la modification parfois brutale du régime alimentaire, les conditions d'environnement changent aussi fortement : modification des conditions d'habitat (stabulation, température, luminosité...), du rythme d'alimentation, des paramètres sociaux... Dans ces conditions, la rentrée à l'étable s'accompagne très généralement, chez la vache laitière en lactation, d'une diminution importante de la quantité de lait produite et de son taux protéique (Burstedt, 1979; Coulon et al., 1986a et b). Ces modifications n'ont cependant pas été observées lorsque la rentrée à l'étable ne s'accompagnait pas de changement du régime alimentaire (Coulon et al., 1987). Elles ne semblent pas dues à un faible niveau d'ingestion de la ration hivernale et peuvent être en partie évitées par un apport supplémentaire temporaire d'aliment concentré (Coulon et al., 1986b). Elles risquent de s'accroître chez des animaux ayant vêlé au printemps ou en été et présentant à la rentrée à l'étable des niveaux de production élevés, supérieurs à ceux observés dans les essais cités précédemment.

Les objectifs de cette étude ont été : 1) de vérifier si les phénomènes observés avec des rations à base de foin ou d'ensilage d'herbe se répètent avec des rations de meilleure valeur alimentaire, à base d'ensilage de maïs (essai 1); 2) de rechercher l'intérêt d'un apport supplémentaire de concentré ainsi que sa répartition la plus efficace pour limiter les diminutions de performances des animaux (essai 2).

\section{MATÉRIEL ET MÉTHODES}

\section{Animaux et alimentation}

Les 2 essais se sont déroulés à l'automne 1986 sur les domaines INRA d'Orcival (essai 1) et de Marcenat (essai 2), entre les semaines -2 et +5 par rapport à la rentrée à l'étable. Dans l'essai 1 , 2 rations de base hivernales ont été comparées : ensilage de maïs (lot $E M$ ) et ensilage de dactyle (lot EH). Dans l'essai 2, réalisé avec une ration à base de foin, l'effet d'un apport d'aliment concentré de $28 \mathrm{~kg}$ supplémentaire à l'apport normal (lot Fo 0 ) a été étudié selon qu'il était distribué au cours de la première semaine de stabulation, à raison de $4 \mathrm{~kg} / \mathrm{vache} / \mathrm{j}$ (lot F40), ou au cours des 2 premières semaines de stabulation, à raison de $2 \mathrm{~kg} / / \mathrm{vache} / \mathrm{j}$ (lot F22). Les 2 lots de l'essai 1 et les 3 lots de l'essai 2 étaient constitués de 8 vaches multipares, appariées sur la base de la production laitière au cours des 2 semaines précédant le début de l'essai, du stade de lactation et de gestation et du poids vif. Les vaches produisaient entre 13 et $26 \mathrm{~kg} / \mathrm{j}$ 
de lait à la rentrée à l'étable $(20 \mathrm{~kg} / \mathrm{j}$ en moyenne) et leur stade de lactation était compris entre 22 et 47 semaines ( 32 en moyenne).

Jusqu'à la date de la rentrée à l'étable (20 octobre dans l'essai 1, 13 octobre dans l'essai 2), tous les animaux ont été conduits de la même façon au pâturage. Avant le début de l'essai, les animaux recevaient un aliment concentré de type production (120 g PDI/UFL), à raison de $1 \mathrm{~kg}$ par tranche de $2,5 \mathrm{~kg}$ de lait au-dessus d'une production de $13 \mathrm{~kg} / \mathrm{j}$. Quinze jours avant la rentrée, les animaux ont commencé à recevoir, à l'étable au moment de la traite, de l'ensilage de maïs (essai 1, lot EM), de l'ensilage de dactyle (essai 1, lot EH) ou du foin de prairie naturelle (essai 2) à raison de $3 \mathrm{~kg} \mathrm{MS} / \mathrm{j}$; cette quantité est passée à $10 \mathrm{~kg} \mathrm{MS}$ le dernier jour de pâturage dans l'essai 2. Dès le premier jour de stabulation (entravée), tous les animaux ont reçu leur ration de base à volonté, en 2 distributions par jour. Dans les 2 essais, les animaux ne sont pas ressortis au pâturage après la première nuit passée à l'étable.

Dans les 2 lots de l'essai 1, l'aliment concentré (de type production) a été distribué à raison de $1 \mathrm{~kg}$ par tranche de $2,5 \mathrm{~kg}$ de lait au-dessus d'une production de $13 \mathrm{~kg} / \mathrm{j}$. La quantité distribuée a été individuellement calculée d'après la production des vaches au cours des semaines -4 et -3 avant la rentrée, et a été maintenue constante pendant tout l'essai. Dans le lot EM, $150 \mathrm{~g}$ d'urée et $700 \mathrm{~g}$ de tourteau de soja tanné ont été substitués au même poids de concentré de production à partir de la rentrée à l'étable, de manière à rééquilibrer la ration de base entre les apports d'énergie et d'azote.

Dans l'essai 2, dans le lot FoO, l'aliment concentré (de type production) a été distribué à raison de $1 \mathrm{~kg}$ par tranche de $2,5 \mathrm{~kg}$ au-dessus d'une production de $8 \mathrm{~kg} / \mathrm{j}$, selon le même principe que dans l'essai 1. Dans le lot F22, dès la veille de la rentrée à l'étable et pendant les 2 premières semaines de stabulation, $2 \mathrm{~kg} / \mathrm{j}$ d'aliment concentré supplémentaires ont été distribués. Dans le lot F40, dès la veille de la rentrée et pendant la première semaine de stabulation, on a distribué $4 \mathrm{~kg} / \mathrm{j}$ d'aliment concentré supplémentaires (Fig. 1).

Dans les 2 essais, tous les animaux ont reçu $150 \mathrm{~g} / \mathrm{j}$ d'un aliment riche en minéraux $(16 \%$ de
Essai I

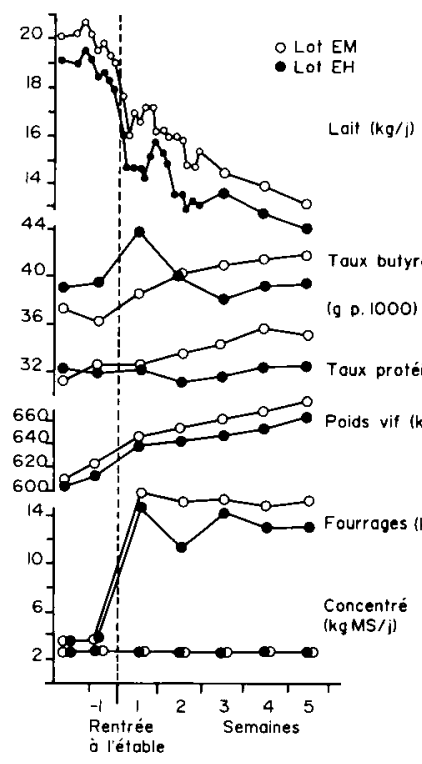

Essai 2

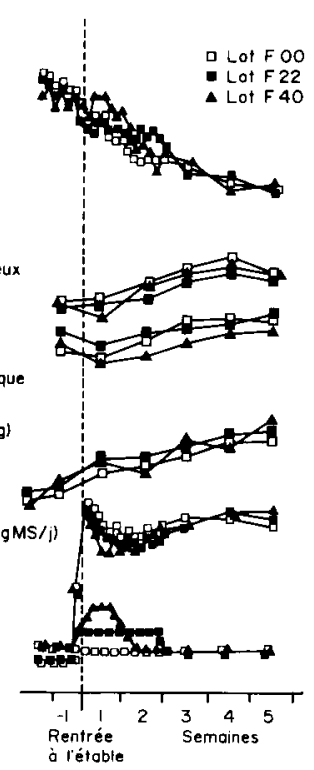

Fig. 1. Evolution des performances des animaux à la rentrée à l'étable. 
Tableau I. Composition et valeur nutritive des aliments.

\begin{tabular}{|c|c|c|c|c|c|}
\hline & \multicolumn{3}{|c|}{ Essai 1} & \multicolumn{2}{|c|}{ Essai 2} \\
\hline & $\begin{array}{l}\text { Ensilage } \\
\text { de maïs }\end{array}$ & $\begin{array}{l}\text { Ensilage } \\
\text { d'herbe }\end{array}$ & Concentré 1 & Foin & Concentré ${ }^{1}$ \\
\hline Teneur en MS $(\%)^{2}$ & 30,2 & 23,2 & 87,0 & 85,5 & 87,0 \\
\hline Matière organique (\% MS) & 95,6 & 90,0 & 92,6 & 91,2 & 92,6 \\
\hline Matières azotées totales ( $\%$ MS) & 8,2 & 17,9 & 19,4 & 15,5 & 19,4 \\
\hline $\begin{array}{l}\text { Cellulose brute (\% MS) } \\
\text { Valeur nutritive }(/ \mathrm{kg} \mathrm{MS})^{3}\end{array}$ & 19,2 & 27,3 & 9,3 & 28,2 & 9,3 \\
\hline UFL & 0,9 & 0,87 & 1,05 & 0,82 & 1,05 \\
\hline PDIN $(g)$ & 52 & 107 & 124 & 96 & 124 \\
\hline PDIE (g) & 66 & 85 & 123 & 93 & 123 \\
\hline
\end{tabular}

${ }^{1}$ Composition (\%) : céréales (40), pulpes de betteraves (30), tourteau tanné (22), mélasse (5), minéraux (3);

${ }^{2}$ Corrigée pour tenir compte des pertes à l'étuve;

${ }^{3}$ D'après INRA (1988).

phosphore, $16 \%$ de calcium et $4 \%$ de magnésium dans l'essai 1; $8 \%$ de phosphore, $10 \%$ de calcium et $8 \%$ de magnésium dans l'essai 2 ).

La composition chimique et la valeur nutritive des aliments sont précisées au Tableau I.

\section{Mesures}

Les quantités individuelles de lait produites ont été pesées tous les jours. Les taux butyreux et protéique individuels ont été mesurés 4 jours la première semaine de stabulation et 2 jours par semaine les autres semaines (essai 1); tous les jours, entre les jours -5 et +5 par rapport à la rentrée, et 4 jours par semaine ensuite (essai 2). Les quantités ingérées ont été mesurées individuellement 5 jours par semaine. Les animaux ont été pesés toutes les semaines (2 jours consécutifs en semaine -1 et +5 ); dans l'essai 2, une notation d'état corporel a été réalisée, par maniement (note de 0 à 5) [Bazin, 1984], en semaine -1 et +6 par rapport à la rentrée à l'étable.

Dans l'essai 2, des prélèvements de sang ont été effectués à la queue des animaux, le matin avant la distribution des aliments, les jours $-5,+2,+5,+9$ et +20 par rapport à la rentrée. Sur le plasma obtenu par centrifugation et conservé au congélateur, on a mesuré, à l'aide d'un appareil de dosage automatique Isamat, la teneur en glucose (méthode de Trinder, 1969) et en acides gras non estérifiés (Agne) [coffret Wako, méthode décrite par Chilliard et al., 1984].

\section{Analyses des résultats}

Les variations de masse corporelle au cours de l'essai ont été estimées entre la première et la cinquième semaine de stabulation, en corrigeant les variations de poids vif pour tenir compte des variations de contenus digestifs, liées aux différences des quantités ingérées entre ces 2 périodes ( $4 \mathrm{~kg} / \mathrm{kg} \mathrm{MS}$ ) [Chilliard et al., 1987].

Les bilans énergétiques et azotés ont été calculés à partir des valeurs des aliments et des besoins des animaux publiés par l'INRA (Vermorel et al., 1987; Vérité et al., 1987). Les bilans énergétiques ont été corrigés pour tenir 
compte des interactions digestives et métaboliques négatives qui s'accroissent avec la proportion d'aliment concentré dans la ration et le niveau alimentaire des animaux (Vermorel et al., 1987).

Le traitement statistique des résultats a été réalisé par analyse de variance à un facteur (lot) des écarts de performances entre la dernière semaine de pâturage et la première semaine de stabulation, en prenant comme co-variable la valeur de la variable étudiée au cours de la dernière semaine de pâturage.

\section{RÉSULTATS}

\section{Production et composition du lait}

Dans l'essai 1, entre la semaine précédant et celle suivant la rentrée à l'étable, la production laitière a diminué fortement, et d'autant plus qu'elle était plus élevée avant la rentrée $(P<0,01)$. Cette décroissance a été plus importante $(P<0,01)$ dans le lot $\mathrm{EH}(-3,8 \mathrm{~kg} / \mathrm{j})$ que dans le lot EM $(-2,9$ $\mathrm{kg} / \mathrm{j})$. Elle s'est poursuivie, mais de manière beaucoup moins forte, jusqu'à la fin de l'essai (Fig. 1). En définitive, par rapport à une production laitière théorique (calculée à partir de la production de la dernière semaine de pâturage et d'une décroissance hebdomadaire de 2,5\%), les animaux des lots EH et EM ont produit, 5 semaines après la rentrée, respectivement 4,2 et $4,4 \mathrm{~kg} / \mathrm{j}$ de moins, et le déficit cumulé a atteint, sur les 5 semaines, respectivement 135 et $124 \mathrm{~kg}$ de lait. Exprimés en lait à $4 \%$ de matières grasses, ces écarts sont moins importants dans le lot EM que dans le lot $\mathrm{EH}$, en raison de l'augmentation progressive importante du taux butyreux dans ce lot à la rentrée à l'étable $(+4,7 \mathrm{~g}$ $\%$ entre la dernière semaine de pâturage et la cinquième semaine de stabulation, contre $+1,2 \mathrm{~g} \%$ dans le lot $\mathrm{EH}, P<0,05$ ). Le taux protéique du lait n'a pratiquement pas varié au cours de l'essai dans le lot EH (+ $0,2 \mathrm{~g} \%$ entre la dernière semaine de pâturage et la cinquième semaine de stabulation), alors qu'il a continuellement augmenté dans le lot $\operatorname{EM}(+2,2 \mathrm{~g} \%)$. En définitive, les quantités de matière utile produites ont diminué de manière identique dans les 2 lots entre la dernière semaine de pâturage et la première semaine de stabulation $(-180 \mathrm{~g} / \mathrm{j})$, mais plus fortement dans le lot EH que dans le lot EM entre la dernière semaine de pâturage et la cinquième semaine de stabulation (respectivement -455 et $-347 \mathrm{~g} / \mathrm{j}, P<0,01$ ).

Dans l'essai 2, entre la semaine précédant et celle suivant la rentrée, la production laitière a diminué d'autant moins fortement que les apports de concentrés en première semaine étaient plus importants (respectivement $-1,9,-1,2$ et $-0,6 \mathrm{~kg} / \mathrm{j}$ dans les lots F00, F22 et F40, $P<0,01$ ). Cette décroissance a commencé la veille de la rentrée, c'est-à-dire dès que le foin a été consommé en quantité importante (9 kg MS/j) [Fig. 1]. Pendant la même période, le taux butyreux n'a pratiquement pas varié $(-0,2 \mathrm{~g} \%$ ), tandis que le taux protéique diminuait de $1,1 \mathrm{~g} \%$, de manière voisine dans les 3 lots.

La diminution des apports de concentré dans les lots F40 et F22 en seconde et en troisième semaine de stabulation a entraîné une diminution parallèle de la production de, respectivement, 1,8 et $1,4 \mathrm{~kg} / \mathrm{j}$. De ce fait, les productions laitières des 3 lots ont été voisines en fin d'essai (Fig. 1), de même que les productions de matière utile. 
Quantités d'aliments ingérées et bilans énergétique et azoté

Dans les 2 essais, les quantités ingérées de fourrage ont augmenté fortement à la rentrée à l'étable et ont été maximales dès la première semaine de stabulation. Dans l'essai 1, les quantités ingérées d'ensilage de maïs ont été en moyenne supérieures de $2 \mathrm{~kg} \mathrm{MS} / \mathrm{j}$ à celles d'ensilage d'herbe. Dans l'essai 2, les différences d'apport de concentré au cours des 2 premières semaines de stabulation entre les 3 lots n'ont entraîné que de faibles écarts de consommation de foin $(-1,3 \mathrm{~kg}$ MS entre les lots F0O et F40 en première semaine, pour une différence de quantité de concentré ingérée de $+3 \mathrm{~kg}$ MS) [Fig. 1]. En définitive, les animaux ont ingéré, au cours des semaines 3 à 5 de stabulation, entre 2,5 (lot EH) et $2,7 \mathrm{~kg} \mathrm{MS} / 100 \mathrm{~kg}$ de poids vif (lots EM, F00, F22 et F40), pour des apports de concentré représentant entre 15 et $19 \%$ de la ration totale.

Les bilans énergétiques ont toujours été positifs au cours des 5 semaines de stabulation. En moyenne, ils ont varié de $+2,4 \mathrm{UFL} / \mathrm{j}$ dans le lot $\mathrm{EH}$ à $+4,4 \mathrm{UFL} / \mathrm{j}$ dans le lot EM.

Les bilans azotés ont été largement positifs tout au long de l'essai dans tous les lots (respectivement $+595,+600,+615$, +391 et $+315 \mathrm{PDI} / \mathrm{j}$ en moyenne sur les 5 semaines de stabulation dans les lots F0O, F22, F40, EH et EM).

\section{Poids vif, état corporel et composition du sang}

Dans les 2 essais, la rentrée à l'étable s'est accompagnée d'une augmentation de poids vif de 16 à $31 \mathrm{~kg}$ selon les lots, probablement due à un accroissement du poids du contenu digestif, lié au change- ment de la nature de la ration de base. Au cours de la période de stabulation, cette augmentation de poids s'est poursuivie de manière importante et voisine dans tous les lots $(+19$ à $+30 \mathrm{~kg}$ entre la première et la cinquième semaine de stabulation). Les variations estimées de masse corporelle ont été de, respectivement, 0,92 et $1,08 \mathrm{~kg} / \mathrm{j}$ dans les lots $\mathrm{EH}$ et $E M$, et de $1,12,0,92$ et $1,06 \mathrm{~kg} / \mathrm{j}$ dans les lots $F 00$, F22 et F40. Cette augmentation de masse corporelle a été d'autant plus importante que les animaux était moins forts producteurs à la rentrée $(P<0,01)$, mais elle n'a pas été liée à la diminution de production laitière au cours de l'essai. Elle n'a été liée au bilan énergétique moyen que dans l'essai 1 (Fig. 2), où le coût énergétique de $1 \mathrm{~kg}$ de gain de masse corporeile (estimé par la pente de l'axe principal de la régression entre ces 2 variables [Chilliard et al., 1987] a été de 2,4 UFL.

Dans l'essai 2, où l'état corporel des animaux avait été apprécié en début et en fin d'essai, celui-ci s'est accru, en moyenne dans les 3 lots, de 0,2 point entre la dernière semaine de pâturage et la sixième semaine de stabulation.

Dans les 3 lots, la rentrée à l'étable s'est accompagnée d'une légère et non significative augmentation des teneurs en glucose $(+1,6 \mathrm{mg} / 100 \mathrm{ml}, P>0,05)$ et

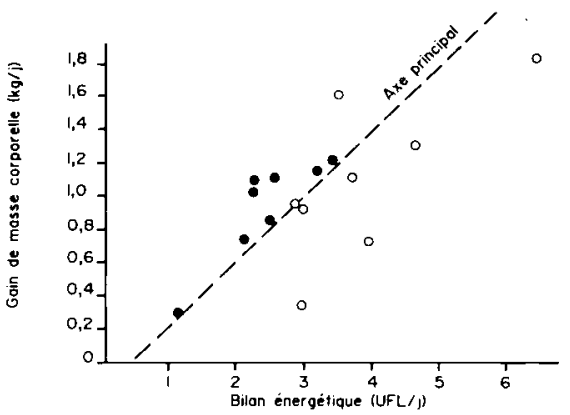

Fig. 2. Liaison entre le gain estimé de masse corporelle et le bilan énergétique (essai 1). $O$ lot $\mathrm{EM} ;-$ lot EH. 
d'un maintien des teneurs en AGNE à une niveau faible $(0,13 \mathrm{mM} / \mathrm{l})$.

\section{DISCUSSION ET CONCLUSION}

Ces 2 essais confirment que, à la rentrée à l'étable, les vaches laitières en lactation sont capables d'ingérer rapidement des quantités élevées de fourrage, quelle que soit la nature de ce fourrage. Cependant, malgré cette augmentation rapide des quantités ingérées, déjà observée dans les essais antérieurs (Burstedt, 1979; Coulon et al., 1986a et b), les productions de lait et de matière utile ont diminué fortement, même avec la ration à base d'ensilage de maïs pour laquelle les apports énergétiques calculés étaient nettement supérieurs aux besoins (bilan calculé en première semaine de stabulation de 2,9 UFLj, en prenant une production laitière égale à celle de la dernière semaine de pâturage). Cette diminution a été moins importante avec la ration à base de foin, mais ce résultat est difficile à interpréter, dans la mesure où cette ration n'a pas été utilisée dans le même essai que les rations à base d'ensilage d'herbe ou de maïs. Les résultats de l'essai 2 confirment, d'autre part, l'effet spécifique favorable des rations à base d'ensilage de maïs sur la composition chimique du lait, comparativement aux rations à base d'ensilage d'herbe (Hoden et al., 1985).

Cependant, contrairement à ce que nous avions observé antérieurement (Coulon et al., 1986a), la diminution de la production laitière ne semble pas signifier que les apports énergétiques totaux disponibles à la rentrée à l'étable aient été surestimés en raison d'une diminution de la digestibilité des aliments. Dans ces essais, i) s'agit plutôt d'une modification brutale de la répartition de l'énergie entre la production laitière et la reconstitution des réserves corporelles. En effet, d'une part, la rentrée à l'étable s'est accompagnée d'une légère augmentation des teneurs plasmatiques en glucose et d'un maintien à un faible niveau des teneurs en AGNE, d'autre part, la masse corporelle estimée s'est accrue au cours de l'essai. Si l'on admet que la reprise de $1 \mathrm{~kg}$ de masse corporelle nécessite 4,5 UFL (Chilliard et al., 1987), les bilans énergétiques «nets" ainsi calculés deviennent négatifs et varient, en moyenne sur les 5 semaines de stabulation, entre - $1 \mathrm{UFL} / \mathrm{j}$ dans le lot EM et -2 UFLj dans le lot F00. Ce résultat signifie que les besoins des animaux ont été surestimés ou que les apports de la ration ont été sous-estimés, hypothèses contradictoires avec celles proposées auparavant (Coulon et al., 1986b). Cependant, ces résultats sont à interpréter avec prudence : en effet, les calculs de bilans énergétiques ont pu être biaisés pour plusieurs raisons. En premier lieu, l'estimation des variations des contenus digestifs est certainement très approximative : ces contenus ne sont en effet vraisemblablement pas stabilisés une semaine après le changement de régime. Or, cette correction affecte fortement les bilans énergétiques calculés : une erreur de $10 \mathrm{~kg}$ de contenus entraine une modification de 1,6 UFL du bilan moyen sur l'essai. D'autre part, dans l'essai 2, les variations d'état corporel sont faibles comparativement aux gains de poids, environ 3 fois moins importantes que les équivalents généralement retenus (35 kg de poids vif par point de note d'état) [Remond et al., 1988]. Enfin, il est possible que la composition de ce gain soit particulière et ne nécessite pas autant d'énergie que nous l'avons supposé (4,5 UFL/kg), ce qui semble confirmé par l'estimation faite dans l'essai 1 (2,4 UFL/kg). 
En définitive, si la rentrée à l'étable s'accompagne toujours d'une diminution importante de la production laitiere, les essais connus concernant cette période ne permettent pas de conclure sur les causes et les conséquences de ces variations, ni sur les moyens de les éviter. Ainsi, dans l'essai 2, si un apport supplémentaire important de concentré à la rentrée a permis de limiter la diminution de production laitière, cet effet ne s'est pas maintenu audelà de la période d'apport supplémentaire, contrairement aux observations antérieures (Coulon et al., 1986a). Dans l'avenir, il serait nécessaire : 1) de travailler sur une période plus longue pour mieux apprécier les variations de poids et d'état corporel ainsi que la persistance de production après la rentrée; 2) d'étudier le partage de l'énergie à la rentrée à l'étable et les causes éventuelles de sa modification; 3) de réaliser des mesures spécifiques (digestibilité de la ration) afin de mettre en évidence d'éventuelles modifications de la digestion à cette période.

\section{RÉFÉRENCES}

Bazin S. (1984) Grille de Notation de l'Etat d'Engraissement des vaches Pie Noires. Ed. ITEB, 149, rue de Bercy, 75595 Paris Cedex 12

Burstedt E. (1979) Some aspects of the nutritive value of grazed grass and carry over effects in the autumn. European grazing workshop, Lelystad, avril 1979

Chilliard Y., Bauchart D. \& Barnouin J. (1984) Determination of plasma non-esterified fatty acid in herbivores and man : a comparison of values obtained by manual or automatic chro- matographic, titrimetric, volumetric and enzymatic methods. Reprod. Nutr. Dev. 24, 469-482

Chilliard Y., Remond B., Agabriel J., Robelin J. \& Vérité R. (1987) Variations du contenu digestif et des réserves corporelles au cours du cycle gestation-lactation. Bull. Tech. CRVZ Theix, INRA 70, 117-131

Coulon J.B., Garel J.P. \& Hoden A. (1986a) Production laitière en zone de montagne : évolution à la rentrée à l'étable. Bull. Tech. CRVZ Theix, INRA 63, 21-24

Coulon J.B., D'Hour P., Garel J.P. \& Petit M. (1986b) Performances des vaches laitières à la rentrée à l'étable à l'automne : influence du type de ration de base et de la quantité de concentré offerte. Ann. Zootech. 35, 37-48

Coulon J.B., Petit M. \& D'Hour P. (1987) Evolution de la production et de la composition du lait autour de la rentrée à l'étable : influence du changement d'environnement. Ann. Zootech. 36, 207-214

Hoden A., Coulon J.B. \& Dulphy J.P. (1985) Influence de l'alimentation sur la composition du lait. 3. Effets des régimes alimentaires sur les taux butyreux et protéique. Bull. Tech. CRVZ Theix, INRA 62, 69-79

INRA (1988) Alimentation des Bovins, Ovins et Caprins. INRA Publications, 147, rue de I'Université, 75007 Paris

Remond B., Robelin J. \& Chilliard Y. (1988) Estimation de la teneur en lipides des vaches laitières Pie Noires par la méthode de notation de l'état d'engraissement. INRA Prod. Anim. 1, 111-114

Trinder P. (1969) Determination of glucose in blood using glucose oxydase with alternative glucose acceptor. Ann. Clin. Biochem. 6, 24-27

Vérité R., Michalet-Doreau B., Chapoutot P., Peyraud J.L. \& Poncet C. (1987) Révision du système des Protéines Digestibles dans l'Intestin (PDI). Bull. Tech. CRVZ Theix, INRA 70, 1934

Vermorel M., Coulon J.B. \& Journet M. (1987) Révision du système des Unités Fourragères (UF). Bull. Tech. CRVZ Theix, INRA 70, 9-18 